\title{
Calidad de vida
}

\section{Definición y áreas de la calidad de vida en Oncología}

\author{
J. Contreras Martínez
}

El cáncer es actualmente una de los principales prioridades en salud pública por su frecuencia y mortalidad, por la morbilidad de los tratamientos que se emplean y por los costes asociados al mismo'.

Tradicionalmente los resultados de las intervenciones terapéuticas empleadas se han evaluado en términos objetivos en los que se valora únicamente los conceptos clásicos de curación, supervivencia, morbilidad, etc. Pero estos parámetros no tienen en cuenta, en la mayoría de las ocasiones, la opinión del paciente, estableciendo como prioridad fundamental obtener la curación. Cuando la curación no es posible lo que se plantea es el aumentar la supervivencia, pero en muchas ocasiones el beneficio que se consigue en términos de supervivencia es escaso, la mayoría de las veces a base de tratamientos con importantes efectos secundarios y con un elevado coste económico. Esto ha llevado a los médicos en general y a los oncólogos en particular a buscar otros objetivos con el tratamiento que administramos, como podría ser el intervalo libre de enfermedad y el beneficio clínico (tiempo que conseguimos mantener al paciente sin síntomas relacionados con su tumor).

Todo ello nos ha propiciado, desde hace unas décadas, al inicio de un cambio en la mentalidad de los oncólogos clínicos, obligando a introducir en los ensayos clínicos innovaciones metodológicas que incluyan conceptos como calidad de vida y estudios farmacoeconómicos, parámetros ambos significativos a tener en cuenta a la hora de tomar decisiones terapéuticas y conocer la eficiencia de las intervenciones sanitarias, con el objetivo de conseguir el mayor grado posible de salud en el paciente con cáncer ${ }^{2-5}$.

Según la O.M.S la definición de Salud sería un estado completo de bienestar físico, mental y social y no sólo la ausencia de enfermedad ${ }^{6}$. Según el diccionario de la Real Academia de la Lengua el término calidad sería la propiedad o conjunto de propiedades inherentes a una cosa que permiten apreciarla como igual, mejor o peor que las restantes de su especie. El término vida hace referencia a un espacio de

Unidad Multidisciplinaria para el Estudio y Tratamiento del Dolor

Hospital Universitario 12 de Octubre

Madrid tiempo que transcurre desde el nacimiento de un animal o vegetal hasta su muerte ${ }^{7}$. Sin embargo, aunque la mayoría de las personas comprenden intuitivamente las connotaciones que implica la expresión calidad de vida su definición es extremadamente complicada dentro del ámbito de la salud. A menudo el término "calidad de vida" es utilizado por el personal sanitario sin ningún tipo de definición explícita, abarcando elementos muy dispares con objeto de poder cuantificarla. Aunque existen múltiples y variadas definiciones sobre lo que es calidad de vida, afortunadamente cada vez existe un mayor consenso sobre la misma, siendo las más acertadas (desde mi punto de vista) aquellas que hacen referencia a la evaluación subjetiva de la vida entendida como un todo, o la valoración de los pacientes sobre la satisfacción con su nivel de funcionamiento normal comparándolo con el que ellos creían que era posible o ideal 8,9 .

La pregunta que surge inmediatamente es: ¿2Pero como se puede cuantificar algo que por su propia esencia o naturaleza es cualitativo? En los estudios clínicos se realiza mediante una serie de encuestas y formularios que incluyen una serie de preguntas o afirmaciones (ítems) relacionados con diversos aspectos de la vida de los pacientes y que se denominan globalmente cuestionarios de calidad de vida relacionada con la salud (CVRS), o en términos anglosajones Health related Quality of life (HRQoL) $)^{10,11}$. Existen de forma global 3 grupos o tipos de cuestionarios para medir la calidad de vida relacionada con la salud:

- Cuestionarios genéricos: Son aquellos cuestionarios que han sido diseñados para poder emplearse en todo tipo de enfermedades e intervenciones terapéuticas (SF-36, NPH...).

- Cuestionarios específicos para cada tipo de enfermedad o problemas de salud: Son diseñados para valorar grupos específicos de diagnósticos y tratamientos (EORT C-30, Functional Living index, Rótterdam Symptom Checlist, QL index and Cancer Rehabilitation Evaluation System...).

- Cuestionarios específicos de síntomas: Este tipo de cuestionarios no son válidos para valorar la calidad de vida relacionada con la salud directamente, sino sólo aquellos síntomas específicos relacionados con la enfermedad o el tratamiento (Gastrointestinal Symptom Rating Scale4, McGill Questionnaire of Pain...).

Sin embargo no todos los instrumentos de medición de la calidad de vida relacionada con la salud tienen el mismo valor, y precisan de una serie de propiedades para ser aceptables, que se podrían resumir en las siguientes: certeza, vali- 


\section{J. Contreras Martínez}

dez y sensibilidad. A su vez el cuestionario sobre calidad de vida debe ser práctico y fácil de utilizar, apropiado para medir el cambio en la calidad de vida del paciente producido por el problema de salud que se está investigando. En resumen, un cuestionario sobre calidad de vida relacionado con la salud debe cumplir las siguientes características para poder ser aplicable en nuestro medio: fácil de comprender, requerir un tiempo de cumplimiento menor a 10 minutos, no debe tener más de 30 items o cuestiones a responder, debería ser contestado únicamente por el paciente y no por sus acompañantes (familiares o no), con lo cual el paciente debería tener información sobre el proceso que padece, debe ser un cuestionario con una correcta traducción desde el lnglés y debe ser adaptado a la idiosincrasia de la zona donde se va a utilizar, $\operatorname{etc}^{12,13,14}$

Llegado a este punto nos podemos plantear el porqué merece la pena medir la calidad de vida en Oncología, y aunque la repuesta no es fácil ni única, podríamos decir que la medición de la CVRS nos ayudaría a contestar a preguntas como la siguiente: ¿Merece la pena someter a los pacientes a tratamientos oncológicos muy severos, con grandes efectos secundarios y expectativas de conseguir (escasamente) modestas ganancias en la supervivencia? Se puede hacer referencia aquí a la experiencia publicada por Benjamín Movsas ${ }^{15}$ en la que pregunta si preferiría someterse a una Quimioterapia agresiva frente a una más suave por una ganancia de $1 \%$ en la supervivencia, y en función del grupo (control, personal médico o de enfermería, oncólogos...) cambian completamente las respuestas. Por tanto, se puede extraer como conclusión el hecho de que la calidad de vida relacionada con la salud significa diferentes cosas, a diferentes personas en diferentes momentos, y por tanto se recomienda que la prioridad la establezca el paciente. Además un problema añadido a tener en cuenta es el envejecimiento progresivo de la población general, lo cual nos hace diagnosticar tumores cada vez con más frecuencia en pacientes ancianos, en los que se plantean indicaciones "límite" de los tratamientos adyuvantes en Oncología (radioterapia, quimioterapia...). Se establece, por tanto, también la necesidad de valorar la posibilidad de realizar un subgrupo de valoración a la hora de decidir tratamientos agresivos en pacientes de más edad (Oncogeriatría). ${ }^{16}$ Podríamos decir, por tanto, que en general las personas (y más los profesionales sanitarios) comprenden intuitivamente las connotaciones que implica el término Calidad de Vida Relacionada con la Salud (CVRS), sin embargo cuando se revisa la literatura y se consultan los textos dedicados al tema es extremadamente complicado el obtener una definición precisa sobre dicho término, en el que además se utilice un lenguaje común. Pero es que lo profesionales sanitarios a menudo utilizan el concepto de calidad de vida en la práctica clínica diaria sin ningún tipo de definición concreta o explícita, abarcando elementos muy dispares con objeto de poder darle una cuantificación. Además de la asistencia, en trabajos de investigación clínica se realizan con cierta frecuencia referencia a la calidad de vida sin haberla cuantificado de forma correcta y exhaustiva. ¿Qué ensayos clínicos deberían incluir una valoración de la calidad de vida relacionada con la salud? Se puede decir, en términos generales, que no es necesario medir la calidad de vida en ensayos clínicos fase I y II, en donde los objetivos principales de los trabajos son establecer la toxicidad de la intervención terapéutica que se mide y su eficacia. Si es ne- cesario medirla en la mayoría de los estudios fase III y IV, en donde se valora la supervivencia de dicha intervención terapéutica. Por ejemplo, cuando se comparan 2 esquemas de tratamientos paliativos, o cuando se comparan 2 tratamientos con similares expectativas de eficacia, o cuando hay una mejora clara de resultados con un brazo de tratamiento pero a expensas de una gran toxicidad con el mismo, o cuando se comparan 2 tratamientos de igual eficacia pero con altas tasas de recidivas globales en uno de ellos. En todas estas situaciones sería necesario el cuantificar las diferencias existentes en relación a la calidad de vida en función de la intervención terapéutica empleada.

Cada vez se realizan con más frecuencia ensayos clínicos en los que se valora la calidad de vida con cada uno de los tratamientos que se están valorando?, pero ello no conlleva asociado (en la mayoría de las situaciones) un cambio en la práctica clínica vinculado a los resultados. En un trabajo realizado con más de 100 oncólogos canadienses ${ }^{15}$, el $75 \%$ afirmaba que consideraba que en futuros estudios aleatorizados la CVRS debería ser un resultado a tener en cuenta más importante que la medición de la supervivencia. En ese mismo estudio el $100 \%$ de los encuestados refería que cambiaría su práctica clínica con los resultados que se publicasen en un estudio en el que se valorase la CVRS, sin embargo más del $50 \%$ de dichos oncólogos reconocía que el trabajo relacionado con la valoración de la calidad de vida debería recaer sobre el personal de enfermería, dada la falta de tiempo que padecen, y debido que consideran que su tiempo lo deben emplear en cuestiones más importantes (relegando, por tanto, la calidad de vida a un segundo plano).

Ahora cabría preguntarse: $\dot{2}$ Son comparables los resultados de los estudios de CVRS cuando se emplean distintos instrumentos de medida? La respuesta nos la da Kemmler y cols. en su trabajo publicado en The Journal of Clinical Oncology cuando compara la CVRS en diferentes pacientes oncológicos cuantificada mediante 2 instrumentos de medida distintos (EORTC QLQ C-30 y FACT-G) y obtenía aspectos marcadamente diferentes con ambas escalas, a pesar de presentar algunas áreas de solapamiento entre ambas ${ }^{17}$.

Respecto a la calidad de vida en el paciente oncológico sometido a radioterapia quiero destacar aquí el trabajo realizado por los Servicios de Oncología Radioterápica de los Hospitales Ramón y Cajal, Puerta de Hierro y Central de la Defensa, con 55 pacientes que reciben tratamiento radioterápico con diferentes diagnósticos tumorales, en los que se valoró la ansiedad, depresión, calidad de vida (EORTC QLQ-C30) y escalas de síntomas ${ }^{18}$. En este trabajo se describe una mayor ansiedad en los pacientes al inicio del tratamiento radioterápico, sin embargo no hay cambios valorables sobre la calidad de vida y el grado de depresión en el transcurso del mismo, apreciándose (lógicamente) una mayor puntuación en cuanto a síntomas en las últimas semanas de recibir el tratamiento.

A modo de resumen, se puede decir que los estudios sobre CVRS son un instrumento de medida nuevo a tener en cuenta en la valoración de los resultados obtenidos con los tratamientos oncológicos, en el que se incluye de forma novedosa el punto de vista u opinión del paciente. Pero es que además, los oncólogos clínicos comenzamos a tomar conciencia de que la calidad de vida es un objetivo crítico necesario para orientar al paciente a la hora de tomar una decisión respecto a su enfermedad y su tratamiento (para lo cual 
se requiere por tanto que el paciente cuente con un alto grado de información). Sin embargo, existen todavía muchas reticencias por parte de los profesionales dedicados a la oncología a usar los instrumentos de medida necesarios para medir la CVRS, dentro de la muy ocupada asistencia diaria, dado que aún se consideran los instrumentos de medida complicados, que ocupan mucho tiempo y requieren de una inversión económica (fundamentalmente en personal). Pero es que además, muchos de los cuestionarios que generalmente se usan son traducciones al castellano de origen anglosajón, que no se adaptan muchas situaciones particulares de nuestra idiosincrasia. A pesar de todo, se debe decir que la CVRS nos muestra, al contrario de lo que puedan interpretar los profesionales sanitarios, que algunos pacientes pueden desear obtener un pequeño beneficio clínico con el tratamiento a pesar del costo del mismo (en términos económicos o de toxicidad).

Podemos concluir diciendo que las únicas personas que pueden evaluar en profundidad la decisión sobre su calidad de vida (o de muerte según las circunstancias) son aquellas que se enfrentan a ella, y que en el planteamiento del tratamiento de pacientes con tumores avanzados se debería dar igual importancia a mejorar tanto la cantidad como la calidad de vida, pues es tan importante añadir años a la vida como vida a los años.

\section{Bibliografía}

1. Aaronson NK. Methodologic issues in assessing the quality of life of cancer patients. Cancer 1991;67:844-50.

2. Yarbro JW. Changing cancer care in the 1999s and the cost. Cancer 1991; 67: 1718-27.

3. Bonsel GJ, Rutten FFH and Uyl de Groot. Economic evaluation alongside cancer trials: methodological and practical aspects. Eur. J. Cancer $1993 ; 29$ (suppl.7) : 10-4.
4. Fernández C. Farmacoeconomía: Estudios de calidad de vida (3ª parte). Farm. Clín. 1990;7;380-9.

5. Sacristán JA, Soto J, Reviriego J, Galende I. Farmacoeconomía: El cálculo de la eficiencia. Med. Clin. (Barc) 1994;103:143-9.

6. González-Barón M, Matín Jiménez M. El dolor en el paciente oncológico. En: Tratamiento sintomático del paciente canceroso. Díaz-Rubio E, Duque Amuso A, García Conde J y col (Ed). Publisalud, S.A. Madrid: 83-103; 1999.

7. Navarro S. Calidad de vida: Parámetros de las ciencias de la salud. NFT 1994; 7:11-2.

8. Bottomley A. The Cancer Patient and Quality of Life. The Oncologist 2002;7:120-125.

9. Bottomley A, Vanvoorden V, Flechtner $\mathrm{H}$, et al. The challenges and achievements involved in implementing Quality of Life research in cancer clinical trials. European Journal of Cancer 39 (2003): 275-285

10. Lugo J, Barroso MC, Fernández L. Calidad de vida y sus instrumentos de medición. Una herramienta en Oncología. Rev. Cubana Oncol. 1996; 12(1).

11. Cohen G. Clinical Research by Community Oncologists. Cancer J. clin. 2003;53:73-81

12. Breslow L. A quantitative approach to the World Health Organization definition of helth: Physical, mental and social wellbeing. Int. J. Epidemiol $1972 ; 1: 347-55$.

13. Sanz O. Valor y cuantificación de la calidad de vida en Medicina. Med. Clin (Barc) 1991; 96:66-9.

14. Velikova G, Stara D, Selby P. Quality of Life Instruments in Oncology. European Journal of Cancer, vol. 35, n 11(15711580); 1999.

15. Movsas B. Quality of Life in Oncology Trials: A clinical guide. Seminars in Radiation Oncology, Vol.13, no 3 (July), 2003;235-247.

16. Maio M, Perrone F. Quality of Life in elderly patients with cancer. Health and Quality of Life Outcomes 2003,1:44.

17. Kemmler $G$ et al. J. Clin. Oncol. $1999 ; 17: 2932-2940$.

18. Cruzado JA. Oncología, 2004; 27 (7): 408-411. 